\title{
Circuit
}

Musiques contemporaines

\section{Créé dans Le Vivier}

\section{Cléo Palacio-Quintin}

Volume 27, numéro 1, 2017

URI : https://id.erudit.org/iderudit/1039677ar

DOI : https://doi.org/10.7202/1039677ar

Aller au sommaire du numéro

Éditeur(s)

Les Presses de l’Université de Montréal

ISSN

1183-1693 (imprimé)

1488-9692 (numérique)

Découvrir la revue

Citer ce document

Palacio-Quintin, C. (2017). Créé dans Le Vivier. Circuit, 27(1), 85-87.

https://doi.org/10.7202/1039677ar

Ce document est protégé par la loi sur le droit d'auteur. L'utilisation des services d'Érudit (y compris la reproduction) est assujettie à sa politique d'utilisation que vous pouvez consulter en ligne.

https://apropos.erudit.org/fr/usagers/politique-dutilisation/
Cet article est diffusé et préservé par Érudit.

Érudit est un consortium interuniversitaire sans but lucratif composé de l'Université de Montréal, l'Université Laval et l'Université du Québec à Montréal. Il a pour mission la promotion et la valorisation de la recherche. https://www.erudit.org/fr/ 


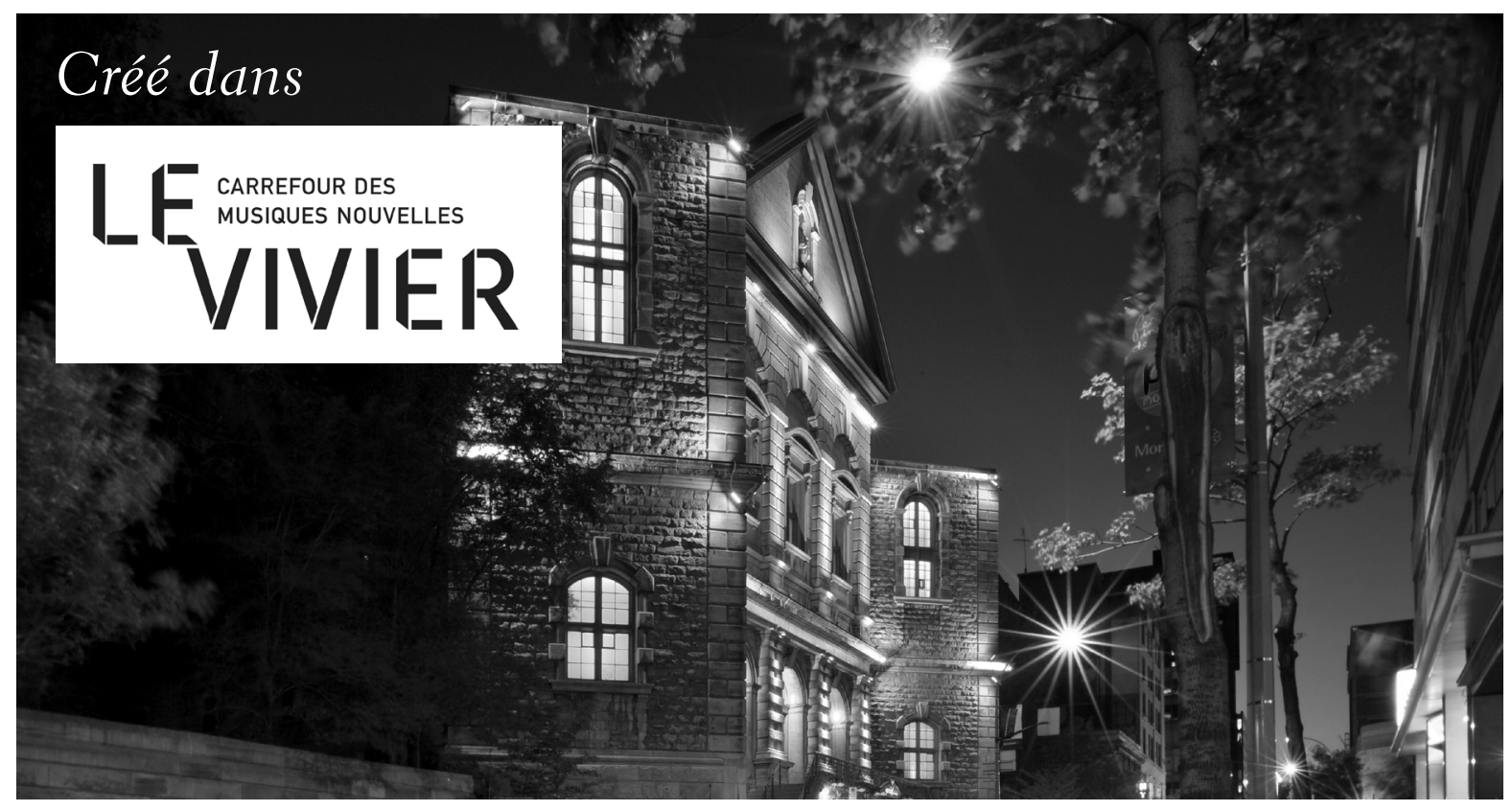

Photo : Le Gesù.

Cléo Palacio-Quintin

Installé dans l'enceinte du Gesù - Centre de créativité depuis janvier 2015, Le Vivier, diffuseur spécialisé et regroupement de 35 ensembles et organismes dédiés aux musiques de création, réside dans ce lieu mythique de la culture afin d'implanter à Montréal un véritable Carrefour des musiques nouvelles. Sa principale mission est de favoriser le développement des musiques nouvelles et d'offrir à tous, par la diffusion d'œuvres de qualité, une porte ouverte sur la culture. La revue Circuit veut mettre en valeur les créateurs et interprètes en présentant une liste des œuvres jouées en création dans le cadre de la série de concerts du Groupe Le Vivier'.

\section{- Saison artistique 2015-2016 -}

Constantinople $\bullet$...et je reverrai cette ville étrange

10 octobre 2015

Navid NAVAB $\bullet$ Ville étrange

Gabriel Dharmoo, Zal Sissokho, Kiya Tabassian • Welcome Calls

Amphithéâtre - Le Gesù

Shawn Mativetsky $\bullet$ Interlude 1

Didem BAșar • Are You "Shur"

NAVID NAVAB $\bullet$ Interlude 2

Didem Başar, Shawn Mativetsky, Kiya Tabassian • Bols 


\section{ZaL Sissoкho $\bullet$ Interlude 3}

Gabriel Dharmoo $\bullet$ Interlude 4

DidEM BAŞAR • Interlude 5

Sandeep Bhagwati $\bullet$ Hark! A Pageant!

ReZA ABAEE $\bullet$ Interlude 6

KIYA TABASSIAN $\bullet$ Interlude 7

KiYa TABASSIAN $\bullet 13-11-15$

Navid Navab, Guy Pelletier $\bullet$ Interlude 8

Gabriel Dharmoo, Zal Sissokho, Kiya Tabassian • Vocal Chords

Nicolas CALOia $\bullet$ Interlude 10

Sound of Montreal : Reza Abaee (gheychak), Didem Başar (qanûn), Nicolas Caloia (contrebasse), Zal Sissokho (kora, voix), Gabriel Dharmoo (voix), Shawn Mativetsky (tabla), Navid Navab (traitement électronique), Guy Pelletier (flûtes), Kiya Tabassian (setar, voix)

21 janvier 2016

Amphithéâtre - Le Gesù

4 février 2016

Amphithéâtre - Le Gesù

8 avril 2016

Amphithéâtre - Le Gesù
Quasar • Série Hommage John Rea - La chèvre de M. Rea

Henry Koch $\bullet$ Atemholen (Take A Breath)

Philippe Macnab-SÉguin • Quatuor de saxophones

Quasar: Marie-Chantal Leclair (saxophone soprano), Mathieu Leclair (saxophone alto), André Leroux (saxophone ténor), Jean-Marc Bouchard (saxophone baryton)

Productions Fiolûtröniq • Souffles électriques

Karen Sunabacka / Malcolm Guite • All Night A Dark Wind Blows

Monique Jean •Volt_\#2

Cléo Palacio-Quintin (hyper-flûtes)

Productions SuperMusique (PSM) et Société de musique contemporaine du Québec (SMcQ) • Série Hommage John Rea - Année Jean Derome - Jeux de pistes

JeAn Derome $\bullet$ Largent

Malcolm Goldstein • Jeu de cartes

Danielle Palardy Roger • Tangram - Orion

Ensemble SuperMusique: Danielle Palardy Roger (direction), Jean Derome (direction, flûte, saxophones), Cléo Palacio-Quintin (flûtes), Lori Freedman (clarinettes), Philippe Lauzier (saxophone soprano), Joane Hétu (voix, saxophone alto), Ida Toninato (saxophone baryton), Craig Pedersen (trompette), Scott Thomson (trombone), Isaiah Ceccarelli (percussions), Corinne René (percussions), Guido Del Fabbro (violon), Josh Zubot (violon), Jean René 
(alto), Jean-Christophe Lizotte (violoncelle), Aaron Lumley (contrebasse), Pierre-Yves Martel (contrebasse), Alexandre St-Onge (basse électrique, électronique), Bernard Falaise (guitare électrique), Vergil Sharkya' (électronique), Martin Tétreault (tourne-disques); Joker: Gabriel Dharmoo, Géraldine Eguiluz, Kathy Kennedy, Elizabeth Lima (voix)

\section{Bradyworks • Instruments of Happiness: 100 guitares électriques}

Tim BRADY • 100 questions, 100 réponses

JoAne HÉTu • Les dentellières

Joane Hétu, Tim Brady (direction)

Instruments of Happiness: Tim Brady, Jonathan Barriault, Simon Duchesne, Marc-Olivier Lamontagne (guitares électriques) et 96 guitaristes invités

\section{Chants libres $\bullet$ The Trials of Patricia Isasas}

\section{Kristin Norderval / NaOmi Wallace • The Trials of Patricia Isasa}

Cristian Germán Gort (direction), Kristin Norderval (soprano - Patricia âgée), Rebecca Woodmass (soprano colorature - Patricia jeune), Dion Mazerolle (baryton - Ramos), Claude Lemieux (comédien - Scilingo), Vincent Ranallo (baryton - Facino), Daniel Pincus (ténor - Juge Brusa), Marie-Annick Béliveau (mezzo-soprano - Gestionnaire Ford), Daniel Binelli (bandonéon), Francis Perron (piano), Nick Danielson (violon), Isabel Castellvi (violoncelle), Pablo Aslan (contrebasse), Marc-Olivier Lamontagne (guitare), Eric Phinney (percussions); Ensemble Kô: Tiphaine Legrand (direction), Annie-Claude Beaudry, Émilie Laforest, Annie Jacques, Ariane Rondeau, Josée Samson, Stéphanie Turcotte (sopranos), Nadine Bourgeois, Annik Geoffroy, Solenn Hellégouarch, Marie-Hélène Rondeau (altos), Paul Bazin, David Désilet, Sylvain Houle, Ruben Shaym-Brutus (ténors), Guillaume Aubertin, Marc-Antoine D’Aragon, Matthew Lane, Gabriel McCann (basses)

\section{Groupe Le Vivier • Cartel 2016 - Le cabaret}

Philippe lauzier, Cléo Palacio-Quintin, Craig Pedersen • Improvisation Combines \#1

Combines: Philippe Lauzier (clarinette basse), Cléo Palacio-Quintin (flûtes), Craig Pedersen (trompette)
9 avril 2016

Espace Aline-Letendre Église du Gesù

Amphithéâtre - Le Gesù

19, 20, 21 mai 2016

Salle Ludger-Duvernay Monument-National

\section{$20^{\mathrm{e}}$ Prix Opus}

L'excellence de la musique

CONCERT DE L'ANNÉE MUSIQUES MODERNE, CONTEMPORAINE

\section{CONCERT DE L'ANNÉE} MONTRÉAL

3 juin 2016

Amphithéâtre - Le Gesù

1. Notez que l'on répertorie ici seulement les œuvres créées lors des concerts inclus dans la saison artistique officiellement présentée par Le Vivier, mais que plusieurs organismes membres présentent aussi d'autres concerts au Gesù au cours de l'année où adviennent également de nombreuses créations. 